\title{
ESTUDO DO COMPORTAMENTO ELETROQUÍMICO DE AMOSTRAS DE AÇO INOXIDÁVEL E DE TI (CP) COM DIFERENTES TIPOS DE REVESTIMENTO
}

\section{STUDY OF THE ELECTROCHEMICAL BEHAVIOUR OF STAINLESS STEEL AND TI (CP) SAMPLES WITH DIFFERENT TYPES OF COATING}

\author{
R. G. CALIXTO ${ }^{1}$, L. CALDEIRA ${ }^{1}$ e J. C. M. da FONSECA ${ }^{2}$
}

${ }^{1}$ Instituto Federal do Sudeste de Minas Gerais - Campus Juiz de Fora, Departamento de Metalurgia, Brasil

2 Faculdade de Engenharia da Universidade do Porto, Departamento de Engenharia Metalúrgica e de Materiais, Portugal

E-mail: r.gomescalixto@gmail.com

article info Article history:

Received 2017-09-04

Accepted 2017-11-20

Available online 2017-12-20
PALAVRAS-CHAVE: Biomateriais; Ensaios Eletroquímicos; Implantes Odontológicos; Osseointegração; Titânio.

KEYWORDS: Biomaterials; Electrochemical Tests; Dental Implants; Osseointegration; Titanium.

RESUMO: $O$ Ti comercialmente puro (cp) tem sido utilizado extensivamente como biomaterial devido a suas características. Outro material amplamente empregado são os aços inoxidáveis, por apresentarem uma grande variedade e um custo acessivel. O objetivo deste trabalho é estudar o comportamento eletroquímico de amostras de Ti e aço inoxidável 316 L através de ensaios eletroquímicos. Os ensaios utilizados para avaliar o desempenho das superfícies foram: Espectroscopia de Impedância Eletroquímica (EIE), Potencial de Circuito Aberto (OCP) e Voltametria Cíclica, realizados em solução de Ringer Lactato. Os resultados obtidos foram analisados a partir do software Gamry Analysis, observou-se que as amostras de aço inoxidável 316 L apresentaram resistência à corrosão inferior às de Ti, nas condições estudadas. Ficou demonstrado que o estado da superficie das amostras é um fator crítico já que dentre as amostras de Ti (cp) aquelas com revestimento apresentaram desempenho eletroquímico superior à usinada.

\begin{abstract}
The commercially pure Ti (cp) has been used extensively as biomaterial because of their characteristics. Another widely used material is stainless steels, because they are widely available and low cost. The objective of this work is study the electrochemical behavior of Ti samples and 316L stainless steel by electrochemical tests. The tests used to evaluate the performance of the surfaces were: Electrochemical Impedance Spectroscopy (EIS), Open Cycle Potential (OCP) and Cyclic Voltammetry, performed in Lactate Ringer's solution. The results obtained were analyzed from the Gamry Analysis software, it was observed that the $316 \mathrm{~L}$ stainless steel samples showed lower corrosion resistance than Ti, under the conditions studied. It was demonstrated that the state of the surface of the samples is a critical factor since among the samples of Ti (cp) those with coating presented best electrochemical performance than machined.
\end{abstract}




\section{INTRODUÇÃOO}

O titânio comercialmente puro tem sido usado extensivamente em aplicações como biomaterial devido a suas características de biocompatibilidade, resistência a corrosão e resistência mecânica (FERREIRA, 2015). Especialmente no campo odontológico tem sido utilizado em implantes permanentes ou provisórios incluindo a modificação de sua superfície para promover a osseointegração. Diferentes técnicas de recobrimento são utilizadas e testadas. Tais técnicas envolvem a anodização em eletrólitos que aumentam a espessura da camada passiva natural e também incorporam elementos que aumentam a adesão e proliferação de células formadoras de tecido ósseo. Diversos estudos têm sido realizados para avaliação da resistência mecânica, da biocompatibilidade e da resistência à corrosão da camada tratada. Entretanto, a espessura e a estrutura de poros da camada passiva possuem um importante papel no processo de osseointegração e apresentam muitos desafios para a redução do tempo de fixação do implante para se colocar a prótese definitiva (ORÉFICE et al., 2006).

Além disso, outro material extensivamente utilizado em aplicações biomédicas apesar da crescente utilização do titânio e suas ligas no mercado é o aço inoxidável, especialmente os da família 316 L. As ligas de aço inoxidável não apresentam a mesma eficiência do que as de titânio, entretanto, essas são ainda são muito utilizadas devido ao preço ser inferior em relação ao titânio, além da grande variedade existente de ligas que podem ser utilizadas como prótese e baixo custo de produção comparado ao titânio. As propriedades dessas ligas devem atender a requisitos como possuir alta resistência mecânica, baixo teor de impurezas e baixa permeabilidade magnética, além de conter uma resistência a corrosão satisfatória quando submetidos a fluídos corpóreos (FERREIRA, 2015).

Esse trabalho visa estudar o comportamento eletroquímico de superfícies de amostras de titânio (com diferentes tipos de revestimento) e aço inoxidável 316 L, ambas fornecidas por um fabricante, cujos processos de revestimentos foram mantidos em sigilo pela empresa. O estudo foi realizado através dos ensaios de Potencial de circuito aberto (OCP), Espectroscopia de Impedância Eletroquímica (EIE) e Polarização Potenciodinâmica Cíclica, com a utilização de um eletrólito que simula os fluídos corpóreos, solução de Ringer Lactato.

\section{DETALHES EXPERIMENTAIS}

\subsection{Preparação das amostras}

As amostras de Ti (cp) e aço inoxidável $316 \mathrm{~L}$ (usinado) em forma de disco, fornecidas pelo fabricante cujos processos de revestimentos foram mantidos em sigilo, foram inicialmente identificadas. Posteriormente, com a utilização de um paquímetro (Mitutoyo, Stainless Hardened. 150x0,05 mm; 6x1/128 in. Precisão: 0,05 mm; Erro: 0,025) e uma régua milimetrada (Precisão: $1 \mathrm{~mm}$; Erro: 0,5 mm) especificou-se as dimensões e estipulou a área de trabalho para cada amostra (Tabela 1). 
Tabela 1 - Dimensões das amostras e área de trabalho proposta

\begin{tabular}{cccc}
\hline Amostra & Diâmetro $(\mathrm{mm})$ & Espessura $(\mathrm{mm})$ & Área de Trabalho $\left(\mathrm{mm}^{2}\right)$ \\
\hline 01.Ti & 6,00 & 3,00 & 16 \\
\hline $02 . \mathrm{Ti}$ & 9,20 & 3,05 & 36 \\
\hline $03 . \mathrm{Ti}$ & 8,00 & 2,00 & 9 \\
\hline 04.Ti & 7,95 & 2,00 & 25 \\
\hline 05.Ti & 8,00 & 2,00 & 25 \\
\hline Inox & 6,00 & 4,00 & 9 \\
\hline Inox FA & 6,00 & 4,00 & 16 \\
\hline
\end{tabular}

Em seguida, com o auxílio de um condutor de prata e um fio de cobre, foi realizado o contato elétrico entra a amostra e o condutor. Após a secagem, verificou o estabelecimento do contato com o uso de um multímetro (Velleman, DVM890) e então para fixar o fio à amostra utilizou-se de uma mistura de cola adesiva de dois componentes (Araldit, Standard) e novamente aguardou a secagem. Finalmente, passou sobre a superfície das amostras um esmalte (verniz) isolando apenas a área de trabalho prevista. Após a secagem foi verificado se a área pressuposta realmente foi à obtida e verificou-se novamente o contato elétrico, sendo que a amostra Inox FA, perdeu o contato elétrico, tornando-se inacessível para a realização dos ensaios. Além disso, as amostras 02.Ti e 04.Ti precisaram ser lixadas em uma região para que o contato elétrico fosse estabelecido e posteriormente verificado, devido supostamente essas duas amostras possuírem uma camada anodizada mais espessa do que as das demais amostras.

\subsection{Preparação do eletrólito}

Como o laboratório o qual os ensaios foram realizados não dispunha no momento do componente lactato de sódio, optou-se por utilizar uma solução de ácido lático $(88,92 \%)$ e hidróxido de sódio sólido para a produção do componente inicial. A solução final almejada foi à solução de Ringer Lactato cuja composição está especificada na Tabela 2. Na tabela apresentada tem se a quantidade para $1 \mathrm{~L}$ de solução, no entanto, no caso do experimento realizado, foram feitas duas soluções de $500 \mathrm{~mL}$, sendo que adotou-se os valores médios de massa para os componentes. 
Tabela 2 - Composição da solução de Ringer Lactato.

\begin{tabular}{cccc}
\hline Componente & $\begin{array}{c}\text { Concentração } \\
(\mathrm{g} / \mathrm{L})\end{array}$ & $\begin{array}{c}\text { Valores } \\
\text { Médios }(\mathrm{g} / \mathrm{L})\end{array}$ & $\begin{array}{c}\text { Quantidade para 500 } \\
\mathrm{mL}(\mathrm{g})\end{array}$ \\
\hline Cloreto de Sódio $(\mathrm{NaCl})$ & 6 & 6 & 3 \\
\hline Cloreto de Potássio $(\mathrm{KCl})$ & $0,27-0,4$ & 0,335 & 0,1675 \\
\hline $\begin{array}{c}\text { Cloreto de Cálcio di-hidratado } \\
\left(\mathrm{CaCl}_{2} \cdot 2 \mathrm{H}_{2} \mathrm{O}\right)\end{array}$ & $0,18-0,29$ & 0,235 & 0,1175 \\
\hline Lactato de Sódio $\left(\mathrm{C}_{3} \mathrm{H}_{5} \mathrm{O}_{3} \mathrm{Na}\right)$ & $2,3-3,3$ & & 1,4 \\
\hline $\mathrm{pH}$ & $6,0-7,5$ & - & - \\
\hline
\end{tabular}

Os dados das duas soluções feitas com a finalidade de serem utilizadas nos experimentos estão descritos na Tabela 3 , onde se pode observar que ambas as soluções ficaram dentro da faixa do $\mathrm{pH}$ previsto.

Tabela 3 - Massas obtidas na preparação das soluções.

\begin{tabular}{|c|c|c|c|}
\hline Componente & $\begin{array}{l}\text { Massa } \\
\text { teórica }\end{array}$ & $\begin{array}{c}\text { Massa pesada - } \\
\text { Sol. } 1\end{array}$ & $\begin{array}{l}\text { Massa pesada - } \\
\quad \text { Sol. } 2\end{array}$ \\
\hline Cloreto de Sódio $(\mathrm{NaCl})$ & $3 \mathrm{~g}$ & $2,9999 \mathrm{~g}$ & $3,0000 \mathrm{~g}$ \\
\hline Cloreto de Potássio $(\mathrm{KCl})$ & $0,1675 \mathrm{~g}$ & $0,1674 \mathrm{~g}$ & $0,1678 \mathrm{~g}$ \\
\hline $\begin{array}{l}\text { Cloreto de Cálcio di-hidratado } \\
\qquad\left(\mathrm{CaCl}_{2} \cdot 2 \mathrm{H}_{2} \mathrm{O}\right)\end{array}$ & $0,1175 \mathrm{~g}$ & $0,1177 \mathrm{~g}$ & $0,1175 \mathrm{~g}$ \\
\hline Hidróxido de Sódio $(\mathrm{NaOH})$ & $0,52 \mathrm{~g}$ & $0,5202 \mathrm{~g}$ & $0,5200 \mathrm{~g}$ \\
\hline Ácido Lático $\left(\mathrm{C}_{3} \mathrm{H}_{6} \mathrm{O}_{3}\right)$ & $1,078 \mathrm{~mL}$ & $1 \mathrm{~mL}$ & $1 \mathrm{~mL}$ \\
\hline $\mathrm{pH}$ & $6,0-7,5$ & 6,22 & 6,6 \\
\hline
\end{tabular}

\subsection{Avaliação da corrosão}

A avaliação da resistência a corrosão foi feita utilizando de uma célula eletroquímica onde foram realizados os ensaios, por meio de um potenciostato/galvanostato/ZRA Gamry Instruments. Foram adotados os mesmos parâmetros para todas as amostras, a única 
diferença foi que no caso da amostra de aço inoxidável o contra eletrodo utilizado foi o de grafite enquanto que nas amostras de Ti foi o de platina. A sequência em que os experimentos foram realizados foi a seguinte. Primeiramente foi determinado o Potencial de Circuito Aberto (OCP) através de um tempo total de 5400 s. Em seguida, realizou-se a EIE, cuja a frequência variou na faixa de $2 \mathrm{mHz}$ a $100 \mathrm{kHz}$. As amostras passaram por um Conditiong de duração de $300 \mathrm{~s}$ e potencial de $-0,7 \mathrm{~V}$ antes de ser iniciado o ensaio de voltametria cíclica o qual teve como limitações inferior e superior, respectivamente os potenciais de $-0,7 \mathrm{~V}$ e $1,5 \mathrm{~V}$ e uma taxa de varredura de $1 \mathrm{mV} / \mathrm{s}$.

\section{RESULTADOS E DISCUSSÕES}

Os resultados foram obtidos através do software Gamry Analysis.

As curvas de potencial versus tempo de imersão em solução de Ringer Lactato para o aço inoxidável 316 L, Titânio (cp) sem revestimento e com revestimento são mostradas no gráfico apresentado na Figura 1. O tempo total de imersão foi de $5400 \mathrm{~s}$.

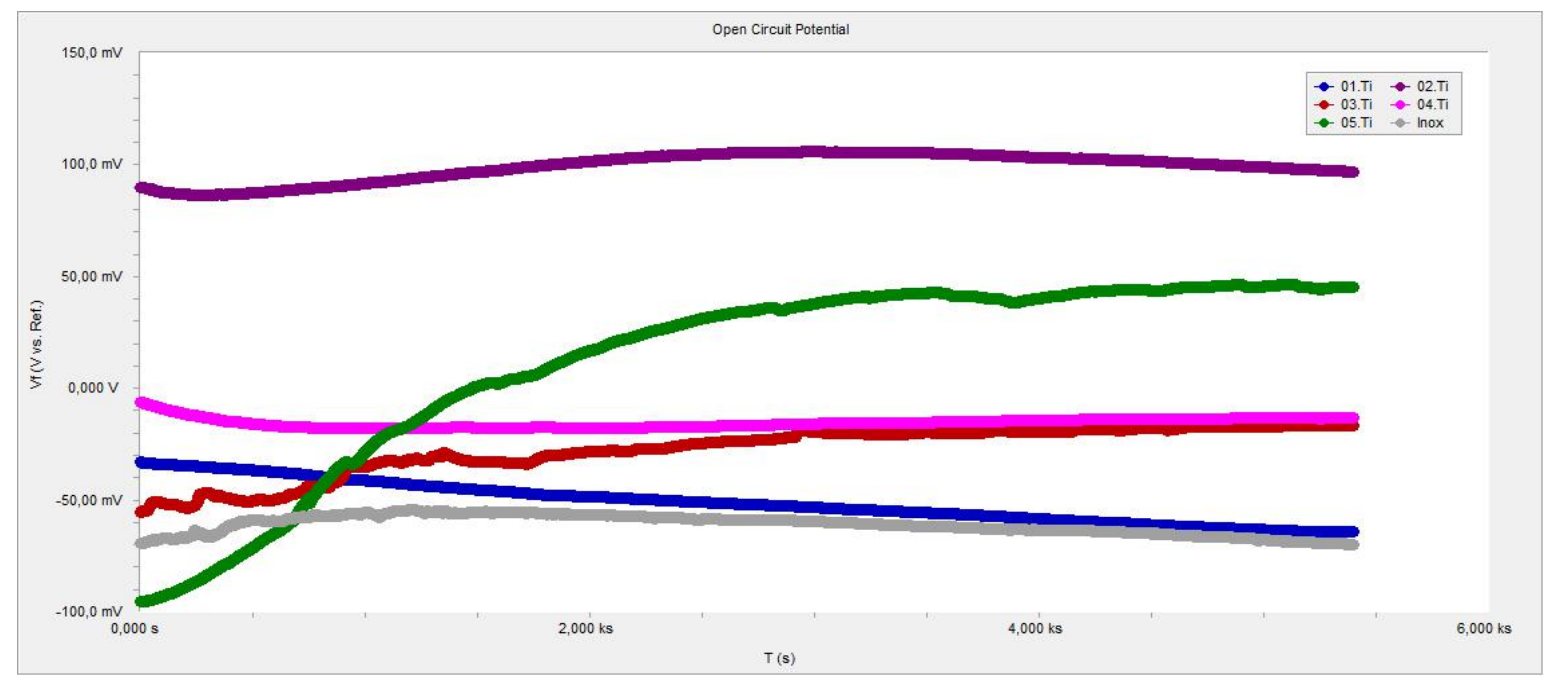

Figura 1 - Gráfico obtido a partir do ensaio de OCP das amostras imersas em solução de Ringer Lactato.

A partir das curvas observa-se que a amostra 02.Ti foi a que apresentou potencial mais nobre em relação as demais amostras. Com o tempo estabelecido do ensaio não se obteve a estabilização das curvas, teve-se apenas uma tendência à estabilização dessas. $\mathrm{O}$ potencial final da amostra 03.Ti que representa a amostra usinada cresceu com o passar do tempo. Alves et al. (2017) em seu trabalho o qual estudou o comportamento de amostras de $\mathrm{Ti}$ (cp) usinadas e produzidas por um tratamento anódico em solução de $\mathrm{NaCl}$, identificaram o mesmo comportamento para a amostra sem revestimento. Os autores propuseram que tal fenômeno ocorreu pelo fato de que com o passar do tempo houve uma diminuição da cinética das reações anódicas devido a formação de uma camada de óxido natural na superfície do titânio que estava em contato com o eletrólito. 
Ao analisar as seis curvas verifica-se que a amostra Inox e amostra 01.Ti apresentaram comportamento menos nobre do que a amostra de $\mathrm{Ti}$ sem revestimento, representada pela curva 03.Ti, entretanto as demais amostras anodizadas obtiveram um potencial mais elevado, implicando que o filme anódico oferece uma melhor proteção quando submetido a esse meio. Portanto, de acordo com esse gráfico em ordem ascendente de nobreza tem-se: Inox, 01.Ti, 03.Ti, 04.Ti, 05.Ti e 02.Ti.

Já a partir do ensaio de EIE obteve-se o gráfico da Figura 2.

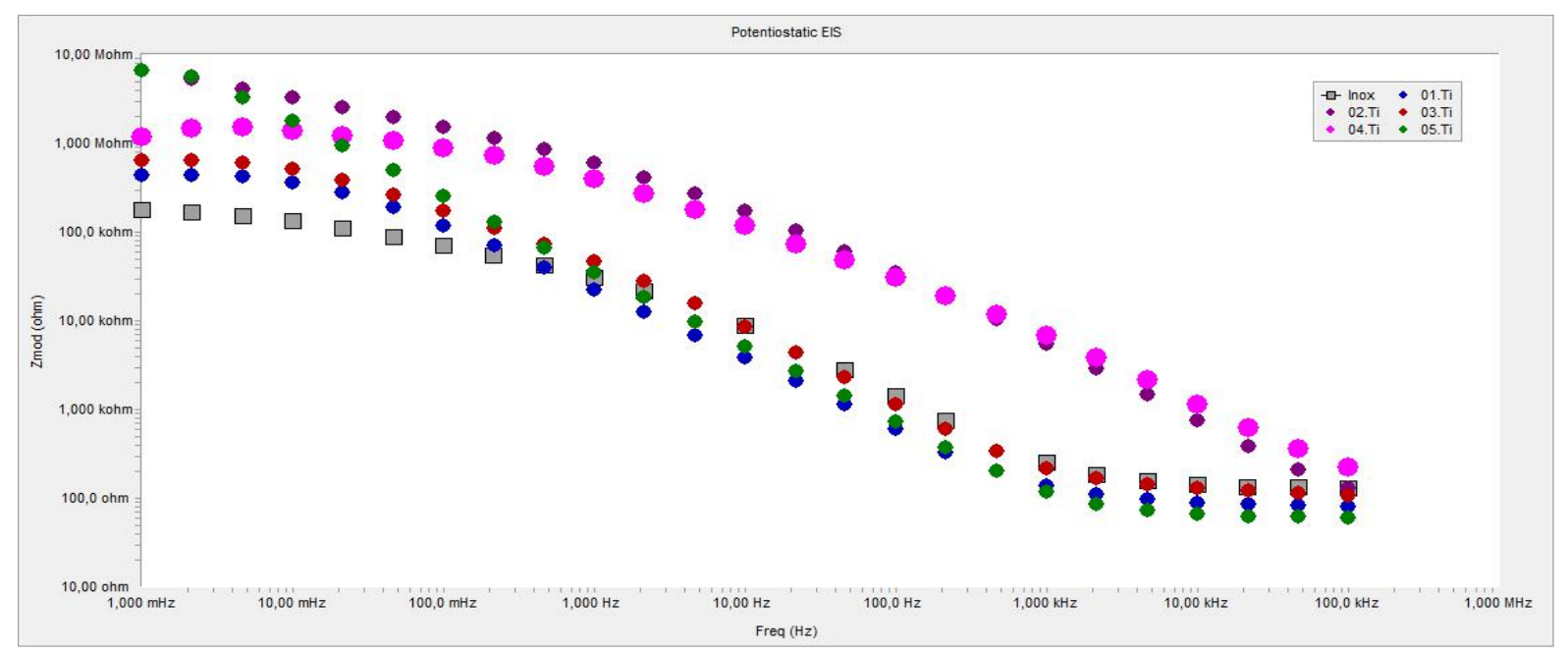

Figura 2 - Gráfico obtido a partir do ensaio de EIE das amostras imersas em solução de Ringer Lactato.

O ensaio de EIE mostrou que a amostra 02.Ti $(6,693 \mathrm{M} \Omega)$ apresentou o maior valor de resistência dentre as amostras, o qual foi muito próximo da amostra 05.Ti (6,582 $\mathrm{M} \Omega$ ) seguido então das amostras 04.Ti, 03.Ti, 01.Ti e Inox, respectivamente. Percebe-se que a amostra Inox foi a que apresentou menor valor de resistência.

Finalmente o ensaio de voltametria cíclica permitiu a obtenção das curvas apresentadas na Figura 3.

A partir do gráfico observa-se que a amostra que apresentou o menor valor de corrente foi a 02.Ti. Em ordem crescente de valores tem-se então as amostras 04.Ti, 05.Ti, Inox, 01.Ti e 03.Ti. Observa-se que a amostra 03.Ti foi a que apresentou maior valor de corrente, sendo que essa representa a amostra usinada e, portanto, não tem nenhuma proteção que impede a passagem da corrente além do filme de óxido natural. Como apresentado no trabalho de Shokouhfar et al. (2012) o qual baseou-se em revestimentos cerâmicos de amostras de Ti utilizando o método de oxidação eletrolítica por plasma e estudou o comportamento eletroquímico em solução de Ringer, os autores apresentam em seus resultados que a película de óxido inserida sobre o metal restringe a adsorção de íons corrosivos, deslocando o potencial superficial e reduzindo a mobilidade carga-portadora na interface metal-eletrólito que por sua vez protege o material da corrosão, sendo o tamanho da 
espessura do revestimento diretamente proporcional a resistência a corrosão. Portanto, a amostra 03.Ti que exibe uma espessura de revestimento mais fina em relação às demais, apresenta uma resistência menor como proposto pelos autores, sendo que as amostras 02.Ti e 04.Ti que apresentaram dificuldade no estabelecimento do contato elétrico supostamente por possuírem uma camada de revestimento mais espessa evidentemente apresentaram uma maior resistência a corrosão.

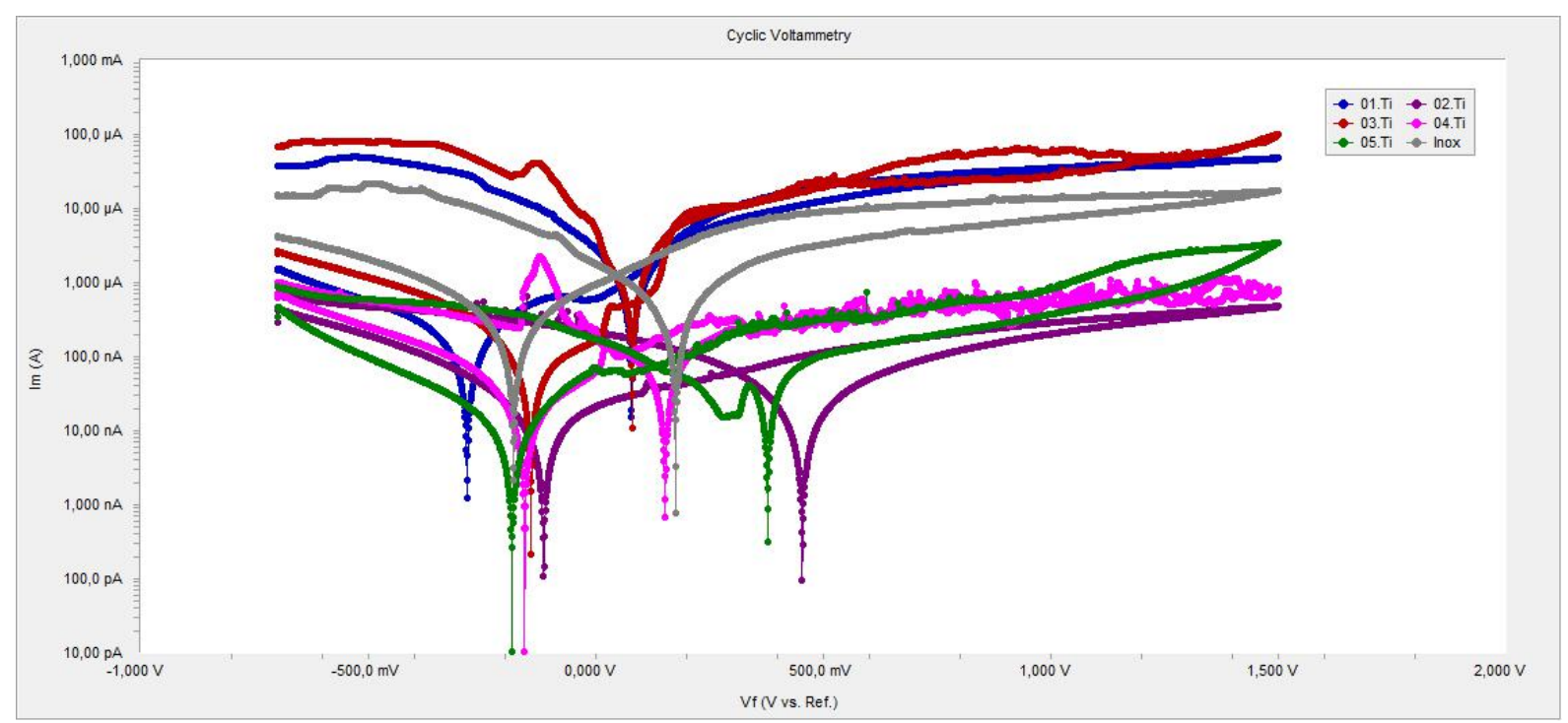

Figura 3 - Gráfico obtido a partir do ensaio de Voltametria Cíclica das amostras imersas em solução de Ringer Lactato.

Essas pequenas oscilações nas curvas, observadas principalmente na curva caracterizada pela cor rosa, representam ruídos que podem ter sido originados por conta de um contato elétrico não satisfatório.

Analisando em conjunto os três ensaios é possível obter a Tabela 4 que deixa evidente o fato de que uma amostra que possui um potencial mais nobre apresenta um filme mais efetivo, possuindo, portanto uma resistência à passagem de corrente elétrica maior e consequentemente uma corrente menor, como é mostrado na tabela. Apesar das divergências entre os resultados obtidos do último ensaio que não seguiram fielmente o que era esperado baseando-se nos dois primeiros, esse último ainda assim apresentou resultados próximos do esperado. 
Tabela 4 - Resultados obtidos a partir dos ensaios realizados

Ensaios

\begin{tabular}{|c|c|c|c|}
\hline & Potencial (OCP) & $\begin{array}{l}\text { Resistência } \\
\text { (EIE) }\end{array}$ & $\begin{array}{c}\text { Corrente - Voltametria } \\
\text { Cíclica }\end{array}$ \\
\hline \multirow{6}{*}{$\begin{array}{l}0 \\
0 \\
0 \\
0 \\
0 \\
0 \\
0 \\
0 \\
0 \\
0 \\
0 \\
\stackrel{0}{0} \\
\stackrel{0}{0}\end{array}$} & $\begin{array}{c}\text { Inox } \\
(-69,85 \mathrm{mV})\end{array}$ & $\begin{array}{c}\text { Inox } \\
(0,18 \mathrm{M} \Omega)\end{array}$ & $\begin{array}{c}02 . \mathrm{Ti} \\
(0,46 \mu \mathrm{A})\end{array}$ \\
\hline & $\begin{array}{c}01 . \mathrm{Ti} \\
(-64,03 \mathrm{mV})\end{array}$ & $\begin{array}{c}01 . \mathrm{Ti} \\
(0,43 \mathrm{M} \Omega)\end{array}$ & $\begin{array}{c}04 . \mathrm{Ti} \\
(0,71 \mu \mathrm{A})\end{array}$ \\
\hline & $\begin{array}{c}03 . \mathrm{Ti} \\
(-16,69 \mathrm{mV})\end{array}$ & $\begin{array}{c}03 . \mathrm{Ti} \\
(0,65 \mathrm{M} \Omega)\end{array}$ & $\begin{array}{c}05 . \mathrm{Ti} \\
(3,30 \mu \mathrm{A})\end{array}$ \\
\hline & $\begin{array}{c}04 . \mathrm{Ti} \\
(-13,45 \mathrm{mV})\end{array}$ & $\begin{array}{c}\text { 04. Ti } \\
(1,19 \mathrm{M} \Omega)\end{array}$ & $\begin{array}{c}\text { Inox } \\
(14,41 \mu \mathrm{A})\end{array}$ \\
\hline & $\begin{array}{c}05 . \mathrm{Ti} \\
(45,08 \mathrm{mV})\end{array}$ & $\begin{array}{c}05 . \mathrm{Ti} \\
(6,58 \mathrm{M} \Omega)\end{array}$ & $\begin{array}{c}01 . \mathrm{Ti} \\
(46,42 \mu \mathrm{A})\end{array}$ \\
\hline & $\begin{array}{c}02 . \mathrm{Ti} \\
(96,81 \mathrm{mV})\end{array}$ & $\begin{array}{c}02 . \mathrm{Ti} \\
(6,69 \mathrm{M} \Omega)\end{array}$ & 03.Ti $(95,83 \mu \mathrm{A})$ \\
\hline
\end{tabular}

\section{CONCLUSÕES}

Como apresentado na introdução foi observado que amostra de aço inoxidável 316 L apresenta um desempenho eletroquímico inferior as amostras de Ti. As amostras 02.Ti e 04.Ti que apresentaram dificuldade no estabelecimento do contato elétrico supostamente por conta de possuírem uma camada mais espessa do revestimento foram as que apresentaram as melhores respostas diante dos ensaios. A condição superficial das amostras afeta o comportamento eletroquímico dos materiais testados e, portanto, as amostras com revestimento exibiram desempenho superior.

\section{AGRADECIMENTOS}

Instituto Federal do Sudeste de Minas Gerais - Campus Juiz de Fora, a Faculdade de Engenharia da Universidade do Porto (FEUP), ao Programa de Mobilidade Internacional criado pelo Instituto Federal do Sudeste de Minas Gerais que possibilitou a integração entre as Universidades permitindo o desenvolvimento desse trabalho e fornecendo auxílio financeiro e, aos Professores Lecino Caldeira (IF Sudeste MG) e José Carlos Magalhães Duque da Fonseca (FEUP) pela orientação durante a realização do mesmo. 


\section{REFERÊNCIAS}

ALVES, A. C.; WENGER, F.; CELIS, J. P.; PINTO, A. M.; ROCHA, L. A.; FERNANDES, J. C. S. Corrosion mechanisms in titanium oxide-based films produced by anodic treatment. Electrochimica Acta, v. 234, p. 16-27, 2017.

ANTUNES, R. A. Caracterização do comportamento frente à corrosão de um aço inoxidável austenítico para aplicações biomédicas com revestimentos PVD de TiN, TiCN e DLC. 2006. 332 f. Tese (Doutorado em Ciências na Área de Tecnologia Nuclear - Materiais) - Instituto de Pesquisas Energéticas e Nucleares, Autarquia associada à Universidade de São Paulo. São Paulo, 2006.

FERREIRA, D.F. Estudo da Corrosão de Ligas de Aço Inoxidável e Titânio em Solução de Ringer. 2015. 102 f. Dissertação (Mestrado em Engenharia de Materiais) Universidade Federal de Ouro Preto, Rede Temática em Engenharia e Materiais UFOP - CETEC - UEMG. Ouro Preto, Abril, 2015.

ORÉFICE, Rodrigo Lambert; PEREIRA, Marinalda de Magalhães; MANSUR, Herman Sander. Biomateriais: fundamentos e aplicações. Rio de Janeiro: Cultura Tredica, 2006.

SHOKOUHFAR, M.; DEHGHANIAN, C.; MONTAZERI, M.; BARADARAN, A. Preparation of ceramic coating on $\mathrm{Ti}$ substrate by plasma electrolytic oxidation in different electrolytes and evaluation of its corrosion resistance: Part II. Applied Surface Science, v. 258, p. 2416-2423, 2012. 